\title{
Air flow technique for large scale dispersion and alignment of carbon nanotubes on various substrates
}

James Hedberg

Lifeng Dong

Jun Jiao

Follow this and additional works at: https://bearworks.missouristate.edu/articles-cnas

\section{Recommended Citation}

Hedberg, James, Lifeng Dong, and Jun Jiao. "Air flow technique for large scale dispersion and alignment of carbon nanotubes on various substrates." Applied Physics Letters 86, no. 14 (2005): 143111.

This article or document was made available through BearWorks, the institutional repository of Missouri State University. The work contained in it may be protected by copyright and require permission of the copyright holder for reuse or redistribution.

For more information, please contact BearWorks@library.missouristate.edu. 


\title{
Air flow technique for large scale dispersion and alignment of carbon nanotubes on various substrates
}

\author{
James Hedberg, Lifeng Dong, and Jun Jiao \\ Department of Physics, Portland State University, Portland, Oregon 97207
}

(Received 2 December 2004; accepted 28 February 2005; published online 30 March 2005)

\begin{abstract}
Herein we present a method to disperse and align carbon nanotubes on various substrate surfaces. Using the shear forces associated with a rapidly moving fluid, nanoscale objects were positioned in a direction corresponding to the flow vector of the fluid. Dispersion of carbon nanotubes on microaddressable electrodes via the gas flow method creates opportunities for scaling up the production of nanoscale devices. In this letter, we demonstrate the feasibility of the method and the electrical characterization results obtained after the fabrication of carbon nanotube testing structures. ㅇ 2005 American Institute of Physics. [DOI: 10.1063/1.1897435]
\end{abstract}

The incorporation of carbon nanotube (CNT) technology into mainstream electronic applications is largely dependent on the development of manipulation methods that not only direct the position of the nanotubes in the nanoscale but at the same time are fast, reliable, and scalable. The gamut of all such manipulation schemes can be dichotomized into the following regimes: postgrowth arrangement, in which already grown nanotubes are positioned in the device at some point during the fabrication process, and during growth, or in situ arrangement, where, by various means such as controlled deposition of catalysts, the nanotubes are grown directly in the desired location. ${ }^{1}$ Within the postgrowth manipulation scheme, two major methods have proven to be successful: (a) electromagnetic force manipulation ${ }^{2}$ and (b) mechanical force manipulation. ${ }^{3}$ The content of this letter describes a method developed in our group for CNT manipulation based on the forces associated with a propellant gas. During preparation of this manuscript, Xin and Woolley have presented an apparatus to align CNTs that relies on similar forces but is accomplished by a different design. ${ }^{4}$

Single-wall nanotube (SWNT) soot was purchased from several national suppliers (Bucky USA, CNI, Carbon Solutions, Nanocs, SES Researches). To make the soot usable, a small amount was placed in certain organic solvents (1,2 dichlorobenzene, dimethylformamide, dichloroethane, dichloromethane, ethanol, isopropanol, de-ionized $\mathrm{H}_{2} \mathrm{O}$ ) known to be helpful in the dispersion of SWNTs. ${ }^{5,6}$ Ultrasonic processing in a $40 \mathrm{kHz}$ bath for times ranging from 10 to $120 \mathrm{~min}$ followed. Some samples were also allowed to let stand overnight to be treated by a second ultrasonic bath prior to use.

The apparatus used for the dispersion and alignment is the following: A Pasteur Pipette was used to insert the dispersed CNT solution into the path of a narrow stream of rapidly flowing $\mathrm{N}_{2}$ gas, which in turn directed the solution onto a flat substrate, generally $\mathrm{Si}$ or $\mathrm{SiO}_{2}$. Figure 1 shows a diagram of the apparatus. When the $\mathrm{N}_{2}$ flows near the surface of the substrate, there exist shear forces that produce a torque on the tubes and lead to their alignment and straightening. Such forces are enough to produce, at the very least, general tendencies for an axial direction vector along the flow direction and in many cases, entire fields of parallel CNTs. Naturally, the strength and spatial distribution of the $\mathrm{N}_{2}$ flow will greatly affect the distribution of the tubes. These parameters were altered and optimized by adjusting both the pressure of the gas in line $(5-25 \mathrm{psi})$ and the shape of the nozzle. Figure 2(c) shows a field of well aligned and individually dispersed SWNTs on a Si substrate in comparison to SWNTs dispersed without using the gas flow alignment method, as seen in Fig. 2(a). A large area of uniform coverage can be achieved by using a nozzle or nozzle array that produces a wide stream of parallel gas as shown in Fig. 2(b). Specifically, a multichannel nozzle consisting of small-bore syringes aligned in parallel created a highly controllable stream of flowing fluid. To view a large area in the scanning electron microscope (SEM), a voltage contrast imaging method was used to increase the apparent size of individual nanotubes during imaging, thus enabling SWNTs to be seen even with low magnification. ${ }^{7}$

To broaden the application of this method of dispersion/ manipulation, we chose to disperse several different nanoscale materials, i.e., multi- and single-walled carbon nanotubes, $\mathrm{ZnO}$ nanowires, etc., onto various substrates, some with preexisting electrode structures, others without. Using the gas flow method described above, SWNTs and multiwalled carbon nanotubes were dispersed on various substrate surfaces. These included: bare $\mathrm{Si}, \mathrm{SiO}_{2}, \mathrm{Cu}$ coated $\mathrm{Si}$, and $\mathrm{Si}$ with patterned electrodes. An important feature of this gas flow alignment method is the wide range of substrates that can be used in conjunction with this technique. Essentially, as long as the surface features are stable enough to withstand the moving fluids, and most tested were, any substrate should be suitable for application to this method. A uniform gas flow over the entire substrate allows for the placement of CNTs (and other similarly shaped, i.e., one-dimensional, nanoma-

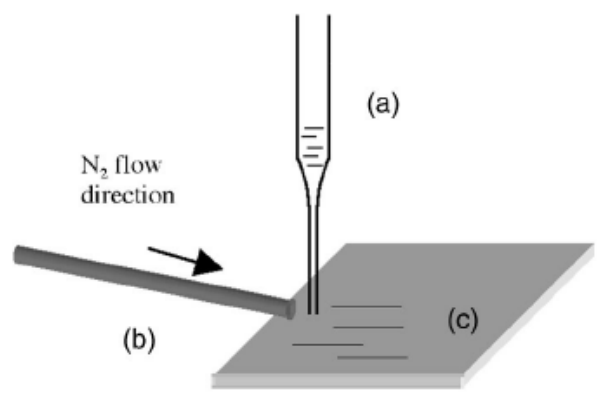

FIG. 1. Diagram of the gas flow alignment setup. (a) Pasteur Pipette to dispense the CNT solution. (b) Nozzle to direct the $\mathrm{N}_{2}$ flow. (c) Si substrate. 

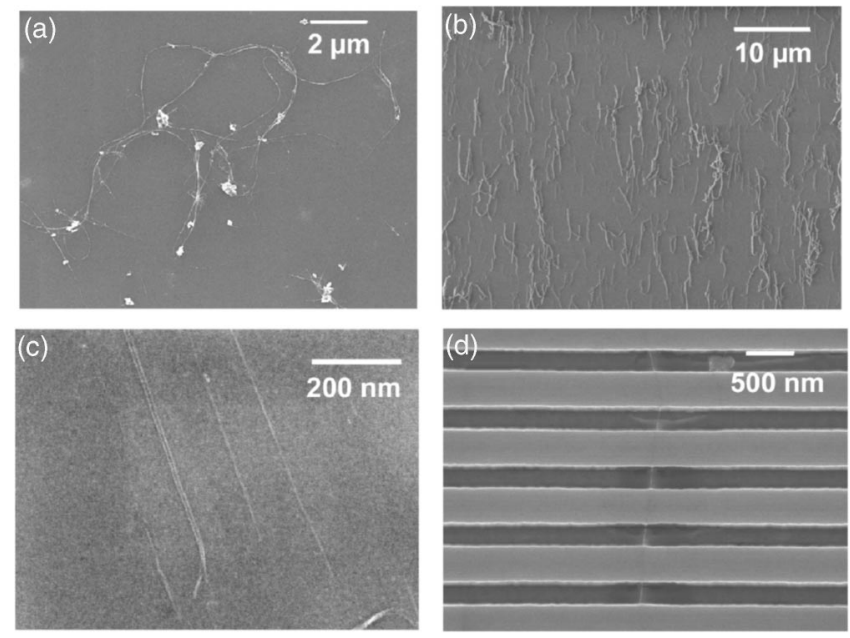

FIG. 2. Comparison of dispersed CNTs (Nanocs in ethanol) (a) without and (c) with gas flow alignment. (b) Large area view of long SWNTs aligned via the multichannel blow method. (Bucky USA in DCB) (d) SWNT aligned across five $300 \mathrm{~nm}$ gaps.

terials) in a regular and purposeful orientation to the substrate. After the dispersion, the samples were characterized in a field emission high-resolution scanning electron microscope (FEI Sirion model). Of immediate notice is the drastic morphological transformation that each individual tube undergoes. Dispersion schemes that do not entail the shear forces associated with the gas flow method tend to leave the CNTs in a very twisted and curved state. Figures 2(a) and 2(b) demonstrate the dramatic difference between tubes without and with gas blow alignment, respectively. Notice that all of the kinks and bends are removed with the gas blow method (b), leaving only straightened CNTs. It has been shown that kinks and bends can lead to anomalous electron transport behavior. ${ }^{8}$ Thus, to obtain the predicted and stable electron transport sought from a CNT, the tube should be as straight as possible. Results from other promising alignment methods, i.e., dielectrophoresis, show tubes that, although generally aligned, still have varying degrees of curvature along the length of the tube that might interfere with electron transport. ${ }^{9}$ It is well known how the growth process can leave a CNT sample quite contaminated with catalyst particles and other undesirables. ${ }^{10}$ An important phenomenon observed in this study is that the gas blow and the pressures

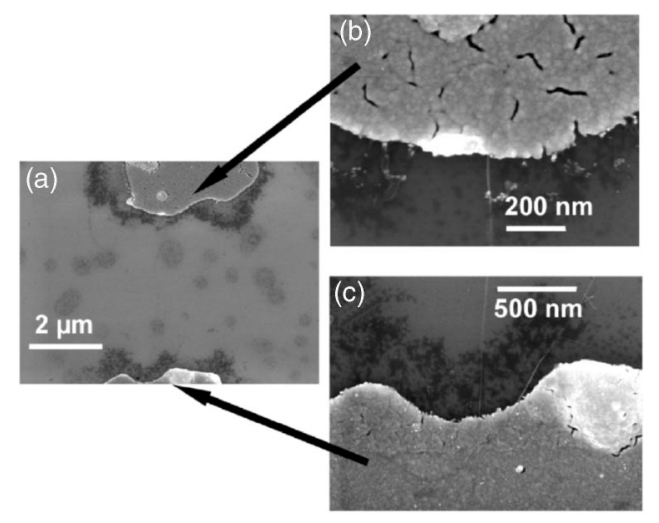

FIG. 3. (a) SEM image shows two Au electrodes separated by a $\sim 4 \mu \mathrm{m}$ gap. (b) and (c) show higher magnification images of the contact points between the CNT (Bucky USA and ethanol) and the upper and lower electrodes, respectively.
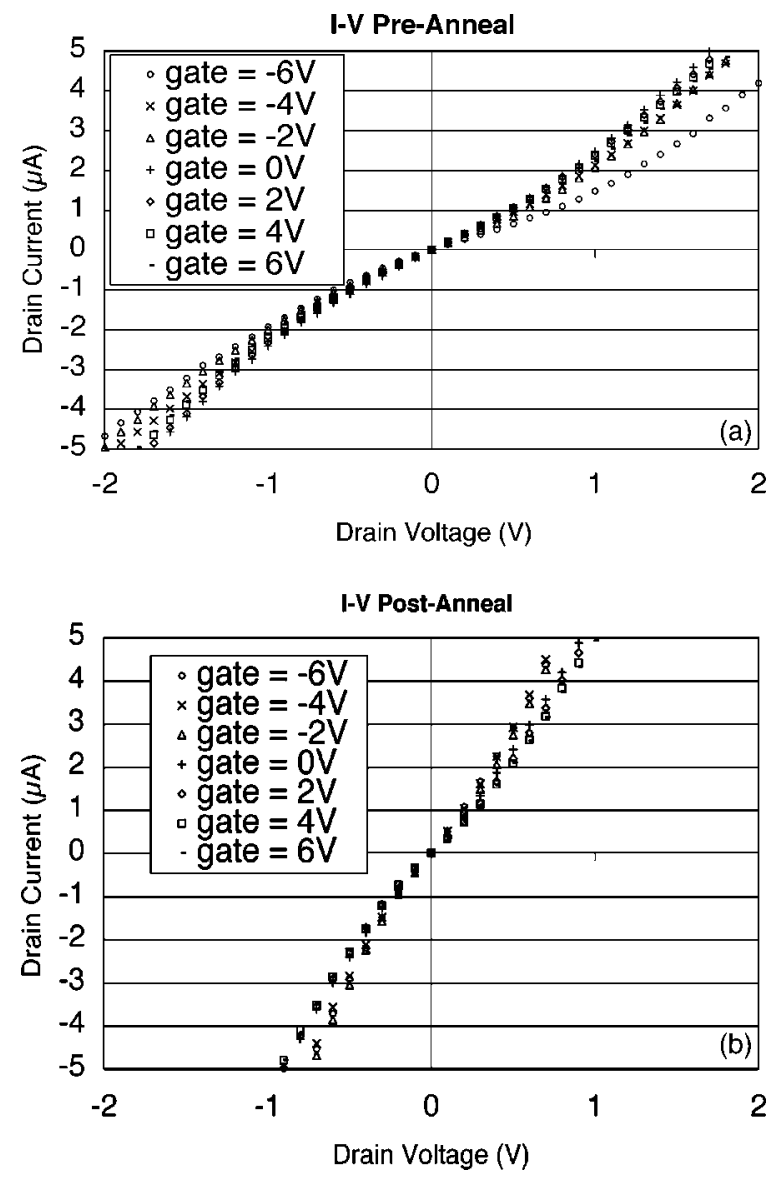

FIG. 4. $I-V$ curves for a SWNT across two Pt electrodes before (a) and after (b) annealing. This nanotube exhibits semiconducting behavior.

associated with the flowing fluids serve to displace unwanted particles from the region of the aligned CNTs. Among the well-aligned SWNTs, very few nanoparticles, such as catalyst particles and carbon debris, were observed in comparison to the original sample. A plausible explanation for this phenomenon is that, in general, the amount of adhesion due to van der Waals interactions increases as the amount of actual contact area between the nanotubes and the substrate surface increases. This amounts to a value for an adhesion potential that varies in proportion to the nanotube contact area. Competing with this value is the kinetic energy of the particle in motion in the flow. Heavier objects with less contact area and higher kinetic energies, namely the undesired catalyst remnants and amorphous bits, will be less likely to be caught by the adhesion potential of the surface. Also, these mechanisms affect the alignment and dispersion of more massive nanomaterials, such as the $\mathrm{ZnO}$ nanowires. Although the nanowires are aligned in the flow field, in this case, they tend not to adhere very well to the surface over which they flow since the van der Waals adhesion potential is much weaker than the kinetic energy.

This technique has proven to be successful for the dispersion of nanotubes over electrodes with both short $(d$ $\sim 200 \mathrm{~nm})$ and large $(d>4 \mu \mathrm{m})$ separation distances; see Figs. 2(d) and 3, respectively. To further test the feasibility of this gas blow technique for the accurate alignment of carbon nanotubes between electrodes, two- and four-point Au electrode configurations were created with standard photolithography. The $\mathrm{N}_{2}$ gas flow method was then used to apply SWNTs and across the gaps, thereby bridging the electrodes 
and making the nanomaterials ready for electronic measurement. It has been elsewhere shown that $\mathrm{Au}$ will make satisfactory electrical contact with SWNTs; ${ }^{11}$ however, this method is applicable to electrodes made from other metals as well.

Using the electrodes created by the alternate production methods, electrode gaps in the order of a few hundred nanometers were made. Nanotubes were dispersed quite easily across this gap. Such results will be useful for a wide variety of applications.

Figure 3 shows an example of a CNT dispersed via the gas flow method across two Au electrodes separated by $\sim 4 \mu \mathrm{m}$. Ever a problem in electrical measurements, the contact resistance between the Au electrode and the CNT must be dealt with. To reduce this contact resistance, thermal annealing at $450{ }^{\circ} \mathrm{C}$ for $30 \mathrm{~s}$ in a $\mathrm{N}_{2}$ (Ar) environment was performed on the samples. ${ }^{12}$ It is expected that the removal of adsorbed molecules affects the tube-electrode interface. ${ }^{13}$ Using a microprobe electrical testing station, standard two probe measurements were obtained. These measurements, as shown, agree with previously published results for nanotube conductivity tests. Establishing the difference between semiconducting and metallic nanotubes was accomplished by applying a gate voltage in a standard field-effect transistor (FET) configuration. Referring to Fig. 4, the results obtained from a testing structure synthesized by the gas blow technique demonstrate the characteristic of the SWNT-FET, where the $I-V$ curves were generated with reference to varying gate voltages, $V_{G}$, range between -6 and $+6 \mathrm{~V}$. Considering the linear region of the $V_{G}=0$ sweep, a value for the resistance of the tube can be calculated. Preannealing resistance was $397 \mathrm{k} \Omega$, which was decreased to $138 \mathrm{k} \Omega$ after thermal annealing. Much of the resistance can be attributed to contact resistance between the CNT and the Pt electrode. This particular nanotube exhibited semiconducting behavior; however, metallic nanotubes were also found among the nanotubes tested.

In conclusion, we have demonstrated an effective means for the placement of carbon nanotubes onto prefabricated electrode structures. This method avoids many of the com- plications presented by electric field alignment and is, in addition, far faster than single tube manipulation by atomic force microscopy or other nanomanipulating devices. Currently we are investigating the analytic aspects of the mechanism responsible for the alignment of nanotubes in a flowing fluid. This method will aid in the development and fabrication of CNT applications by offering an easy and effective means of controlled nanomanipulation.

The authors would like to acknowledge the Intel Corp. for support of this research, the gracious help of Dr. Raj Solanki and the use of his electronic testing facilities at the Oregon Graduate Institute, and Valery Dubin and Ramanan Chebiam for their stimulating conversations. The participation of the undergraduate student for this project is supported by the NSF under Award No. DMR-220926.

${ }^{1}$ H. B. Peng, T. G. Ristroph, G. M. Schurmann, G. M. King, J. Yoon, V. Narayanamurti, and J. A. Golovchenko, Appl. Phys. Lett. 83, 4238 (2003).

${ }^{2}$ Z. Chen, W. Hu, J. Guo, and K. Saito, J. Vac. Sci. Technol. B 22, 776 (2004).

${ }^{3}$ P. A. Williams, S. J. Papadakis, M. R. Falvo, A. M. Patel, M. Sinclair, A. Seeger, A. Helser, R. M. Taylor II, S. Washburn, and R. Superfine, Appl. Phys. Lett. 80, 2574 (2002).

${ }^{4}$ H. Xin and A. T. Woolley, Nano Lett. 4, 1481 (2004).

${ }^{5}$ J. L. Bahr, E. T. Mickelson, M. J. Bronikowski, R. E. Smalley, and J. M. Tour, Chem. Commun. (Cambridge) 2, 193 (2001).

${ }^{6}$ S. Niyogi, M. A. Hamon, D. E. Perea, C. B. Kang, B. Zhao, K. S. Pal, A. E. Wyant, M. E. Itkis, R. C. Haddon, J. Phys. Chem. B 107, 8799 (2003).

${ }^{7}$ T. Brintlinger, Y.-F. Chen, T. Dürkop, E. Cobas, M. S. Fuhrer, J. D. Barry, and J. Melngailis, Appl. Phys. Lett. 81, 2454 (2002).

${ }^{8}$ J. Han, M. P. Anantram, R. L. Jaffe, J. Kong, and H. Dai, Phys. Rev. B 57, 14983 (1998).

${ }^{9}$ X. Q. Chen, T. Saito, H. Yamada, and K. Matsushige, Appl. Phys. Lett. 78, 3714 (2001)

${ }^{10}$ I. W. Chiang, B. E. Brinson, R. E. Smalley, J. L. Margrave, and R. H. Hauge, J. Phys. Chem. B 105, 1157 (2001).

${ }^{11}$ S. Dag, O. Gülseren, S. Ciraci, and T. Yildirim, Appl. Phys. Lett. 83, 3180 (2003).

${ }^{12}$ J.-O. Lee, C. Park, J.-J. Kim, J. Kim, J. W. Park, and K. H. Yoo, J. Phys. D 33, 1953 (2000).

${ }^{13}$ X. Cui, M. Freitag, R. Martel, L. Brus, and P. Avouris, Nano Lett. 3, 783 (2003). 